\title{
Skin Sparing Mastectomy in Locally Advanced Breast Cancer: A Possibility?
}

\author{
Vijayashree Murthy, K. S. Gopinath, Anand Krishna \\ Department of Surgical Oncology, Bangalore Institute of Oncology, Bangalore, India \\ Email: drvijumurthy@gmail.com
}

Received December 9, 2011; revised January 10, 2012; accepted January 22, 2012

\begin{abstract}
Breast cancer is the second most common cause of cancer death in women in India in spite of advances in the adjuvant treatment of breast cancer. Locally advanced breast cancer (LABC) still represents the major presenting picture in many urban and rural institutions in our country. Skin Sparing Mastectomy (SSM) and primary reconstruction is a popular option for patients with breast cancer. There are similar local and distant recurrences in SSM compared to the traditional non-skin sparing mastectomy. A 57-year-old lady presented with a locally advanced left breast cancer. After undergoing neoadjuvant chemotherapy and a PET-CT to accurately detect residual disease in breast and axilla, she underwent skin sparing mastectomy with nipple-areola complex preservation and primary reconstruction with silicon prosthesis implant over a latissimus dorsi myocutaneous flap for primary reconstruction. SSM in LABC has not been reported in the literature so far. This patient is disease free for the past 24 months.
\end{abstract}

Keywords: Skin Sparing Mastectomy; Locally Advanced Breast Cancer; Primary Reconstruction

\section{Introduction}

The incidence of breast cancer is rising in India, with an estimated 80,000 new cases diagnosed annually. The incidence of breast cancer increased by approximately 50\% between 1965 and 1985 and age adjusted rate of breast cancer during 1998-2002 in Bangalore Cancer Registry increased from 18.2 to 25.2 [1]. Much of this increase may be associated with urbanization and improved life expectancy. Locally advanced breast cancer (LABC) is a common presentation in India with patients presenting with large breast masses with skin involvement or matted axillary nodes, which constitutes $40 \%$ of the clinical presentation in medical institutions [2]. Standard treatment would include a metastatic work up followed by neoadjuvant chemotherapy and a traditional mastectomy with or without reconstruction followed by adjuvant treatment protocols (chemotherapy, radiation \pm hormonal and targeted therapy) [3]. Fluorodeoxyglucose Positron emission tomography (FDG-PET) has been used not only for detection and staging, but also chemotherapy response monitoring in breast cancer patients [4,5]. Growing public and surgeon interest in less radical and more cosmetic approaches has led to skin and even further, nipple sparing mastectomy. Toth and Lappert (1991) were the first to describe Skin sparing mastectomy (SSM) which includes resection of the nipple-areola complex (NAC), any existing biopsy scars and removal of the breast parenchyma (with or without an axillary dissection), and this has gained acceptance as an oncologic alternative combined with breast reconstruction [6]. SSM with breast skin preservation has resulted in superior cosmetic appearance [7] however, SSM cannot be performed on all patients with breast cancer especially those with LABC and inflammatory carcinoma.

The current report discusses a patient who presented with left sided LABC and received neoadjuvant chemotherapy followed by FDG-PET assessment to determine the extent of residual disease and then underwent SSM with NAC preservation and primary breast reconstruction using silicon prosthesis over Latissimus dorsi flap. SSM and NAC preservation in LABC has not been reported so far.

\section{Case Report}

In 2007, a 57 year old post menopausal woman, presented with a painless lump in the upper outer quadrant of left breast of five months duration which progressively increased in size. Her past medical history was otherwise unremarkable. General health of the patient was well maintained. Patient's clinical examination revealed a non-tender lump $(6 \times 7 \mathrm{~cm})$ in the upper outer quadrant left breast just lateral to the nipple-areola complex. Overlying skin showed peau d' orange changes $(1 \times 1.2 \mathrm{~cm})$ and axilla had matted nodes $(2 \times 2 \mathrm{~cm})$ in the medial 
group with no other lymphadenopathy. Mammography of the left breast revealed a well-defined dense round mass $(5.6 \times 6 \mathrm{~cm})$ with calcification within it and a tumor-nipple distance of $3.7 \mathrm{~cm}$. A metastatic work up with a chest, abdomen and pelvic CT and bone scan were otherwise normal. FDG-PET was performed which revealed no other disease pick up except the breast and axilla (Figure 1).

After confirming the diagnosis and hormonal receptor status with a breast core biopsy, patient was subjected to 4 cycles of Taxane based chemotherapy. Clinically the lump regressed, skin lesion disappeared, and on mammogram the mass reduced to $3.2 \times 2.2 \mathrm{~cm}$, but still had microcalcifications. Patient then underwent a FDG-PET scan to assess the extent of disease, which confirmed residual uptake in breast and axilla (increased SUV)
(Figure 2). After explaining the potential risk of recurrence to the patient and an additional pre-operative consent for excision of the breast skin and NAC in the event of a positive frozen section or final histopathology, patient was subjected to surgery. A left sided SSM with NAC preserving omega incision was performed to remove the breast and a complete axillary dissection including levels I, II and III completed (Figures 3 and 4). After confirming a negative retroareolar margin on frozen section, primary breast reconstruction was performed by raising a latissimus dorsi myocutaneous flap with silicon textured prosthesis to create a breast mound (Figures 5 and 6). The patient had an uneventful post op period, was discharged 6 days later and resumed normal activities within four weeks. Histopathology revealed an infiltrating duct carcinoma (Grade III), clear margins and

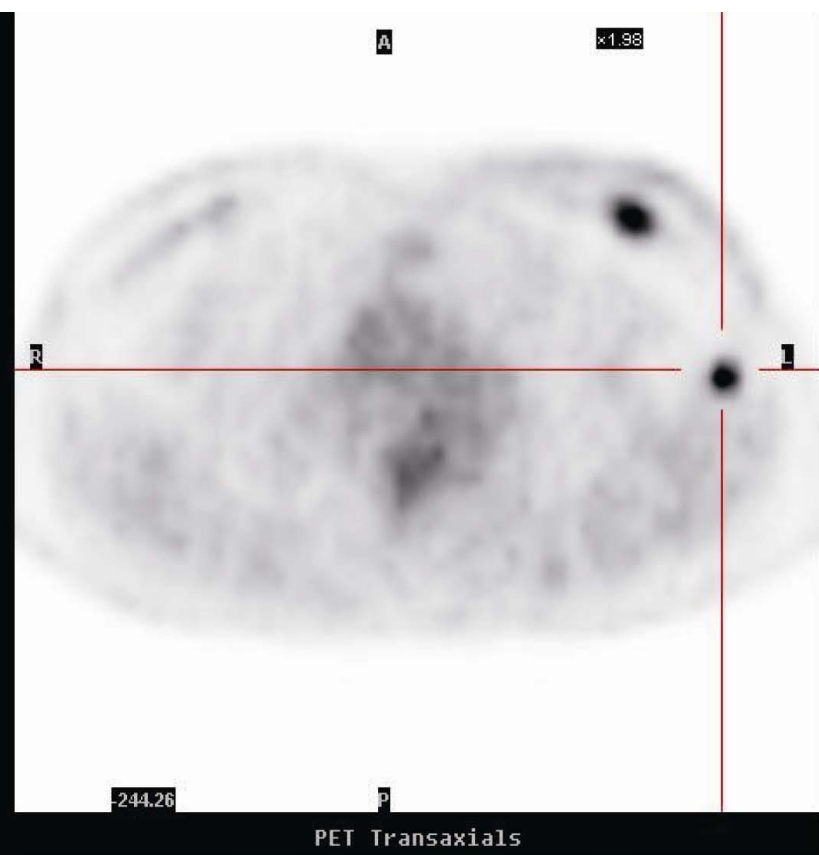

PET Transaxials

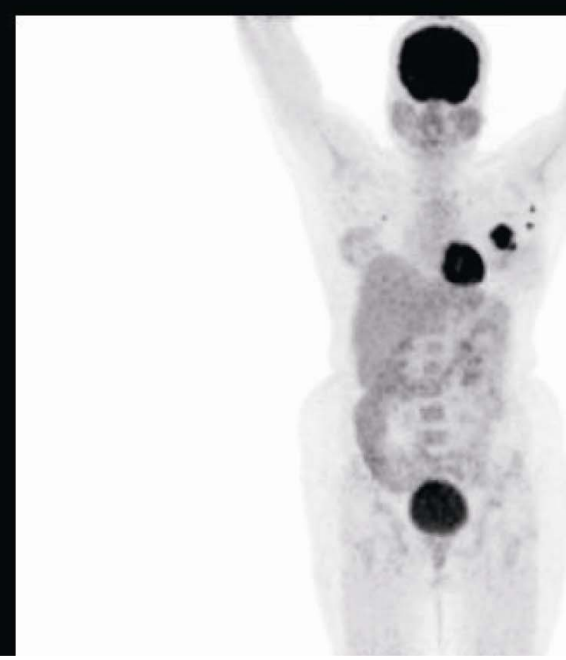

Figure 1. Pre chemotherapy fluorodeoxyglucose PET scan showing malignant disease in left breast and axilla. 


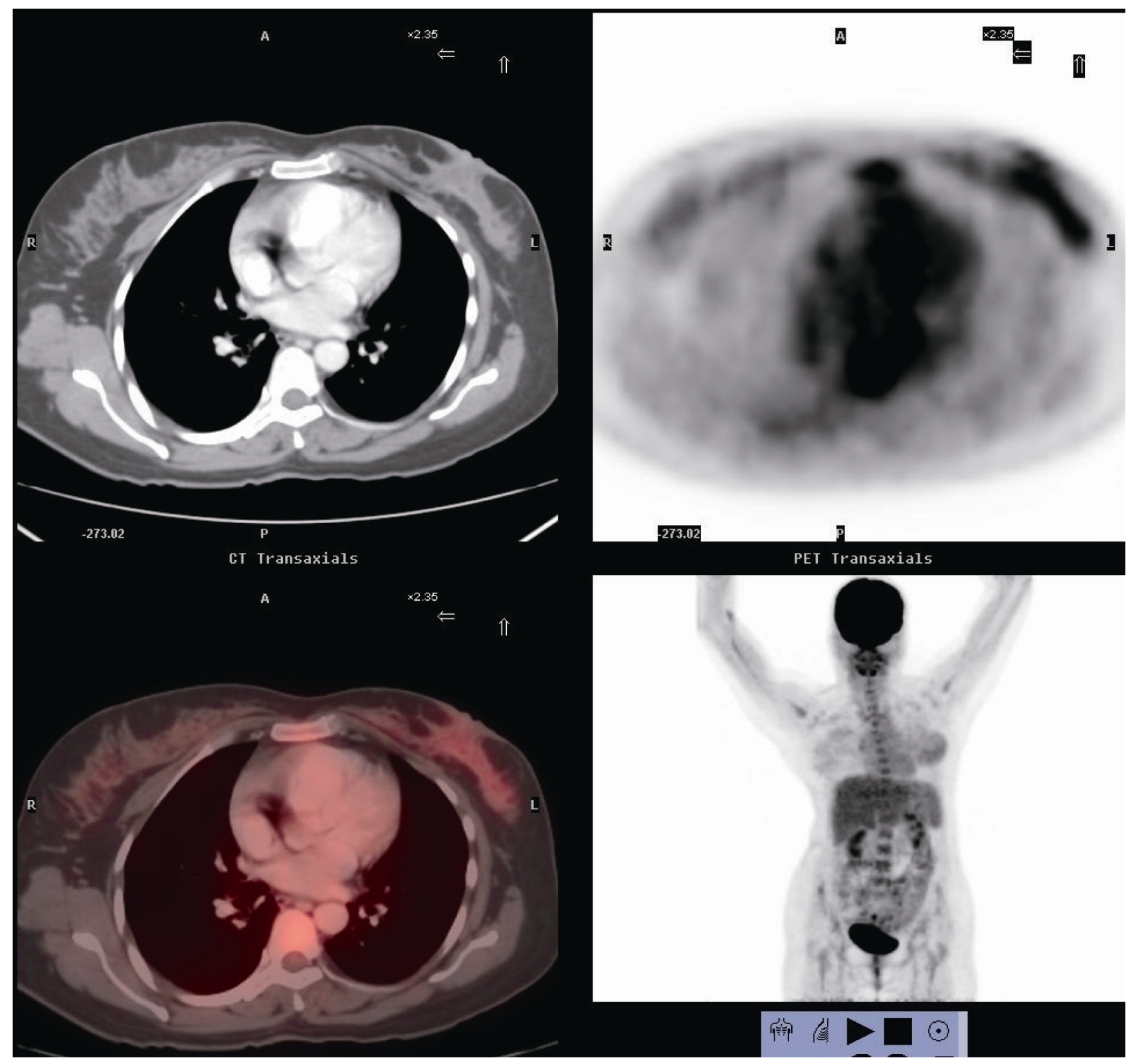

Figure 2. Post chemotherapy fluorodeoxyglucose PET scan showing residual disease in left breast and axilla.

no lymphovascular invasion, with $4 / 24$ nodes positive and ER+/PR+ and HER2 neu negative. Post operatively the patient received chemotherapy and $50 \mathrm{~Gy}$ of external beam radiation therapy in 25 fractions followed by hormones. She remains disease free at 24 months with an excellent performance status.

\section{Discussion}

Patients who present with LABC or multi focal disease require a mastectomy. This not only causes physical but also psychological disfigurement. Numerous studies have documented that the NAC is significant to a woman's body image and quality of life and preservation of breast skin and NAC purports to improve this outcome [8,9]. A popular alternative is SSM which maximizes breast skin preservation and this brings out a superior aesthetic appearance [6]. Unfortunately SSM cannot be done in all patients with breast cancer especially inflammatory carcinoma and LABC. There have been recent publications demonstrating the oncological safety of SSM with NAC preservation in prophylactic and early breast cancer, which improved symmetry with the contra-lateral breast when compared with the traditional non SSM, performed through a wide elliptical incision with a generous resection of breast skin [10]. The aesthetic appearance of the reconstructed breast is largely dependent on the quantity of breast skin that remains after mastectomy. SSM with 

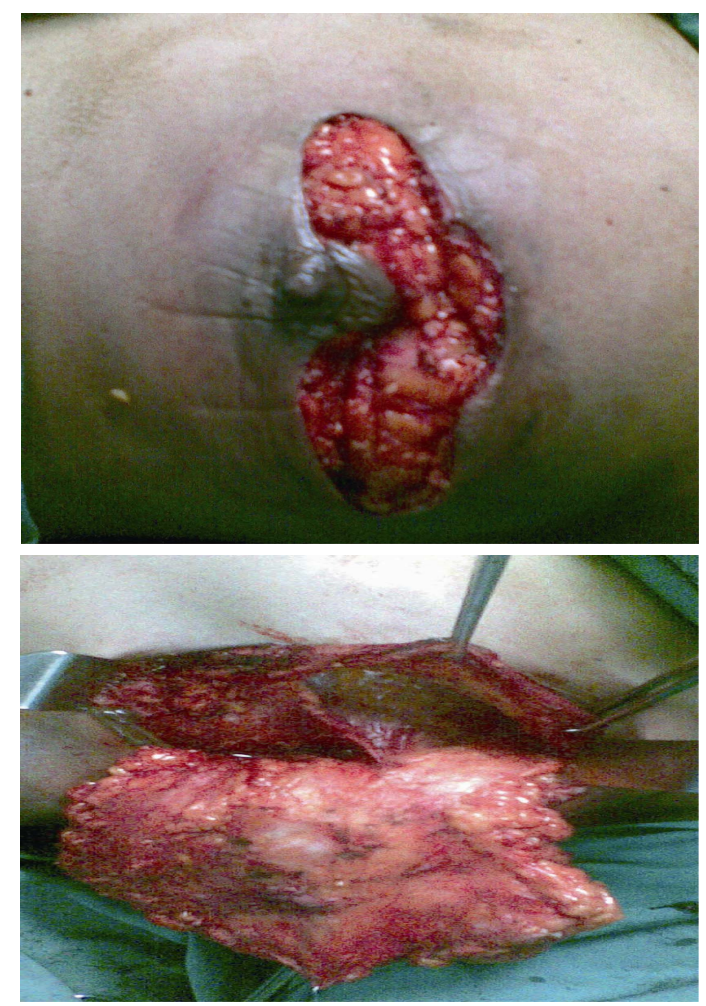

Figure 3. Omega incision with nipple areola complex preservation and raising the breast along with the clavipectoral fascia off the pectoralis major muscle.
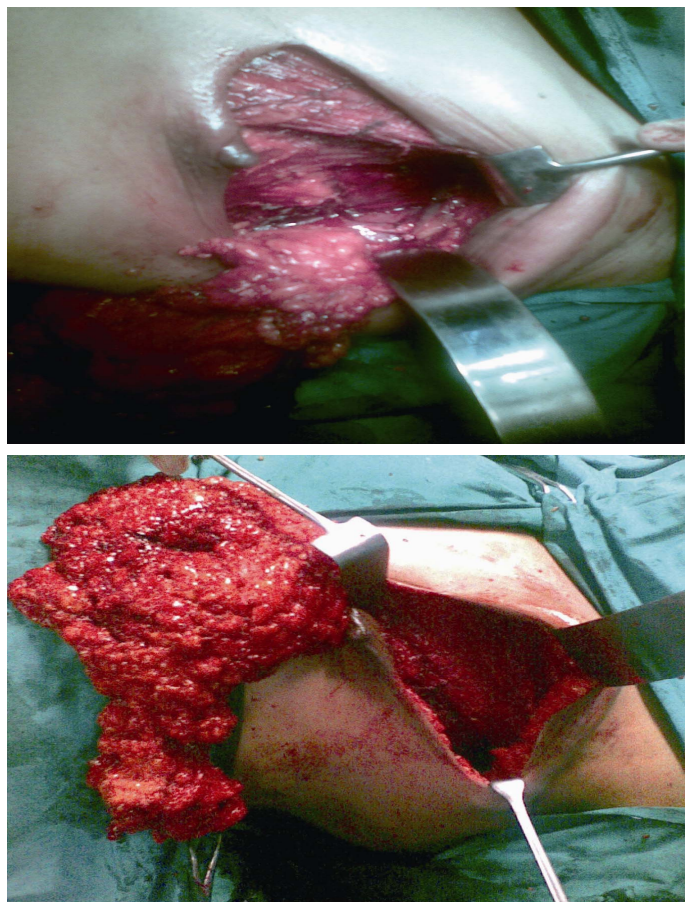

Figure 4. Mastectomy and complete axillary dissection (Levels I, II and III) being completed through the same incision.

NAC preservation offers an opportunity to preserve the native breast envelope for reconstruction without mutila-

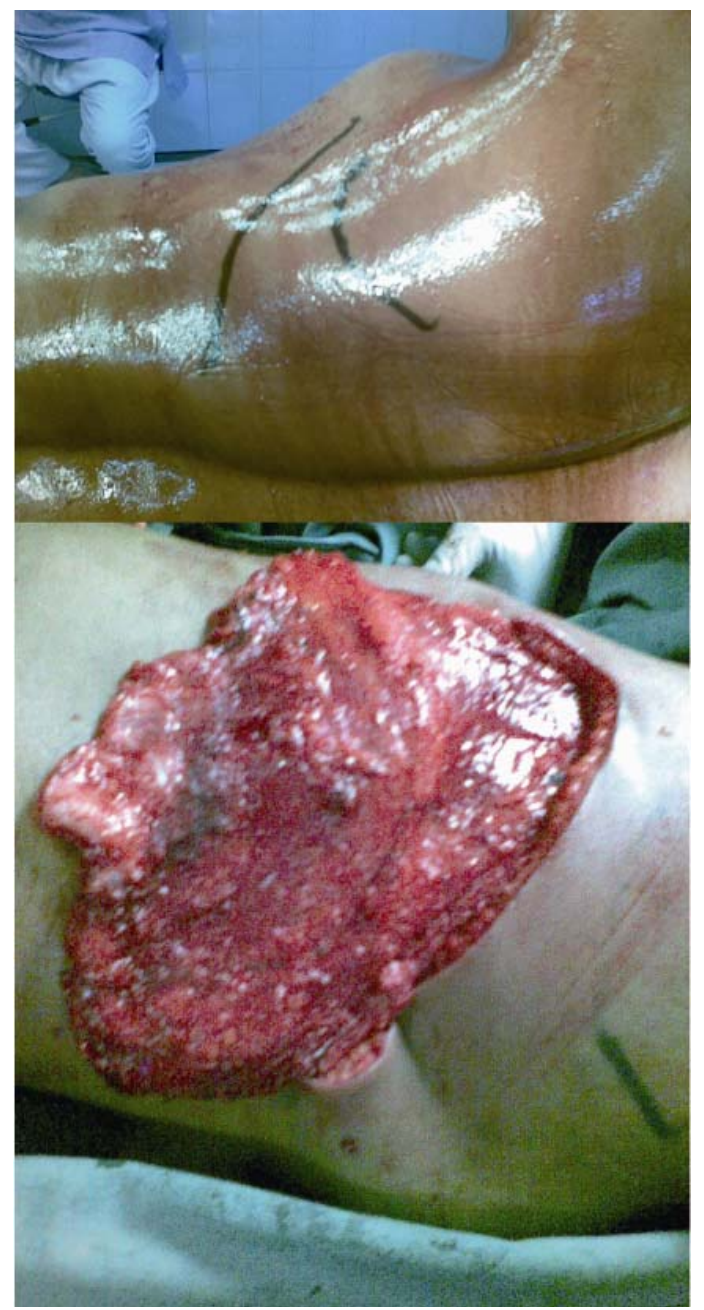

Figure 5. Position and marking of donor site and raising the Latissimus dorsi flap.

tion of the NAC and avoids multiple surgical procedures required for most other forms of reconstruction on the same as well as the contra-lateral breast to achieve symmetry $[11,12]$. SSM with primary reconstruction offers superior cosmetic results to conventional mastectomy with similar local recurrence (LR) rates as seen with early breast cancers [13]. As most LR occurs on the chest wall and skin, the ability to detect them is not impaired and SSM also does not offer any contraindication to adjuvant chemotherapy or radiation. Patients with recurrence after SSM do not fare worse than those who develop recurrence after conventional mastectomy [14]. In a study of 231 mastectomy patients (including 77 SSM and 154 non SSM patients) treated at the Strang-Cornell Breast Center and New York Presbyterian Hospital, Simmons et al reported that the LR rates for SSM and non SSM patients were $3.90 \%$ (3 of 77 patients) and 3.25\% (5 of 154 patients), respectively. The LR free survival at 5 years was $95.3 \%$ for SSM and $95.2 \%$ for non SSM patients $(p=0.28)$. The distant recurrence rates of SSM 


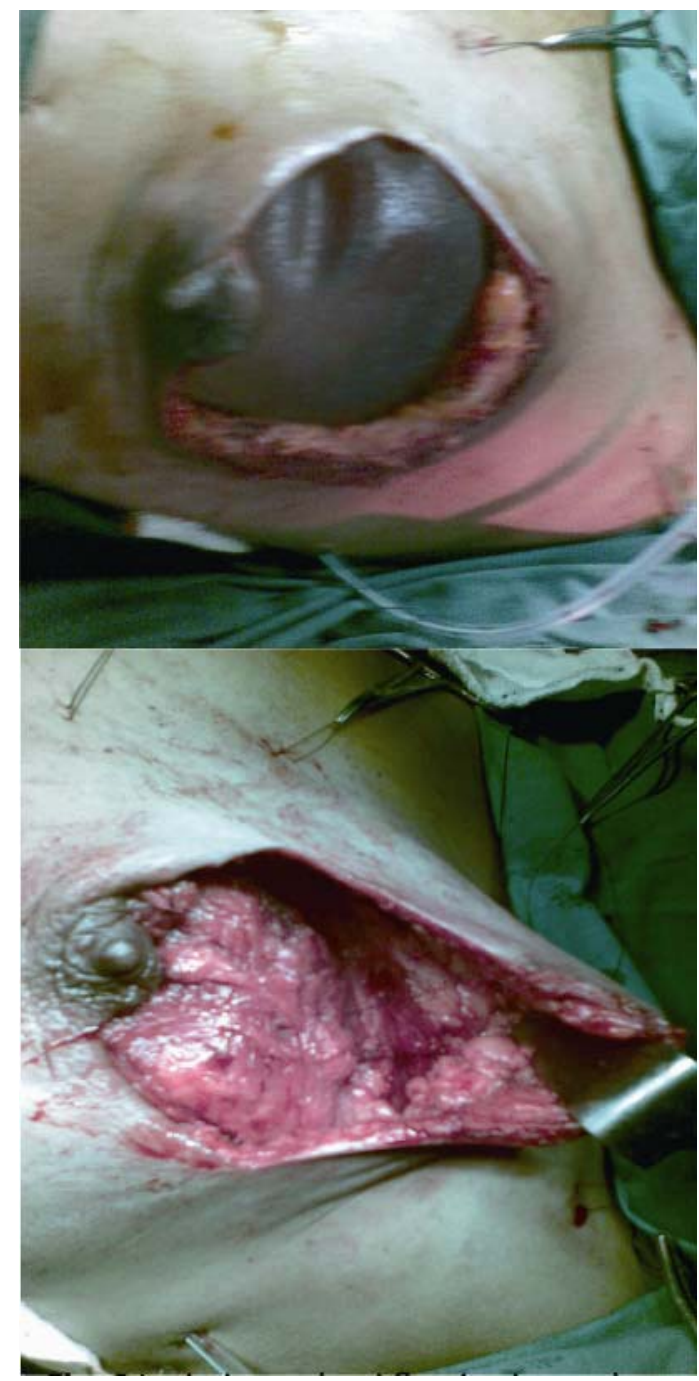

Figure 6. Latissimus dorsi flap in situ and placement of textured silicon implant underneath the flap.

and non SSM patients were 3.9\% (3 of 77 patients) and $3.9 \%$ (6 of 154 patients) respectively, suggesting that mastectomies using the skin sparing technique does not appear to result in any increase in local or distant recurrence in early breast cancers and improve aesthetic results of immediate reconstruction [14].

SSM in LABC has been done in our case as a novel concept and also utilized the evolving role of FDG-PET in assessing and monitoring chemotherapy response in the neoadjuvant setting. This particular case can be considered as an initial attempt to conservative aesthetic surgery in LABC following complete clinical response to neoadjuvant therapy. The patient remains disease free till date. However long-term follow up is required to validate the findings of this study. A team approach to the diagnosis and management of breast tumors is likely to improve the standard of care and patient outcomes. Careful attention to the principles of surgical resection and mar- gin if paid, can improve local and systemic control of disease. Organization and judicious use of this reconstructive technique may permit safe resection of breast tumors possible that were previously fraught with unacceptable patient morbidity. Good oncologic resection with primary reconstruction resulting in an excellent functional outcome and quality of life was achieved in this case.

\section{REFERENCES}

[1] N. S. Murthy, K. Chaudhry, D. Nadayil, U. K. Agarwal and S. Saxena, "Changing Trends in Incidence of Breast Cancer: Indian Scenario,” Indian Journal of Cancer, Vol. 46, No. 1, 2009, pp. 73-74. doi:10.4103/0019-509X.48603

[2] A. K. Goel, V. Seenu, N. K. Shukla and V. Raina, "Breast Cancer Presentation at a Regional Cancer Center," National Medical Journal of India, Vol. 8, 1995, pp. 6-9.

[3] National Comprehensive Cancer Network, "Practice Guide-Lines in Oncology, v.3.2010,” Accessed on 23 November 2010.

http://www.nccn.org/professionals/physician_gls/PDF/bre ast.pdf

[4] K. Dalus, G. Rendl, L. Rettenbacher and C. Pirich, "FDG PET/CT for Monitoring Response to Neoadjuvant Chemotherapy in Breast Cancer Patients,” European Journal of Nuclear Medicine and Molecular Imaging, Vol. 37, No. 10, 2010, pp. 1992-1993. doi:10.1007/s00259-010-1585-Z

[5] D. Groheux, S. Giacchetti, D. Rubello, A. Al-Nahhas and J. L. Moretti, "The Evolving Role of PET/CT in Breast Cancer,” Nuclear Medicine Communications, Vol. 31, No. 4, 2010, pp. 271-273. doi:10.1097/MNM.0b013e3283354cb4

[6] B. A. Toth and P. Lappert, "Modified Skin Incision for Mastectomy: The Need for Plastic Surgical Input in Preoperative Planning," Plastic and Reconstructive Surgery, Vol. 87, No. 6, 1991, pp. 1048-1053. doi:10.1097/00006534-199106000-00006

[7] M. Salhab, W. Al Sarakbi, A. Joseph, S. Sheards, J. Travers and K. Mokbel, "Skin-Sparing Mastectomy and Immediate Breast Reconstruction: Patient Satisfaction and Clinical Outcome,” International Journal of Clinical Oncology, Vol. 11, No. 1, 2006, pp. 51-54. doi:10.1007/s10147-005-0538-1

[8] P. A. Ganz, A. Coscarelli, C. Fred, et al., "Breast Cancer Survivors: Psychosocial Concerns and Quality of Life," Breast Cancer Research and Treatment, Vol. 38, No. 2, 1996, pp. 183-199. doi:10.1007/BF01806673

[9] D. K. Wellisch, W. S. Schain, R. B. Noone, et al., “The Psychological Contribution of Nipple Addition in Breast Reconstruction," Plastic and Reconstructive Surgery, Vol. 80, No. 5, 1987, pp. 699-704. doi:10.1097/00006534-198711000-00007

[10] B. Gerber, A. Krause, M. Dieterich, G. Kundt and T. Reimer, "The Oncologic Safety of Skin Sparing Mastectomy with Conservation of the Nipple-Areola Complex and Autologous Reconstruction: An Extended Follow-Up 
Study," Annals of Surgery, Vol. 249, No. 3, 2009, pp. 461-468. doi:10.1097/SLA.0b013e31819a044f

[11] A. Denewer and O. Farouk, "Can Nipple-Sparing Mastectomy and Immediate Breast Reconstruction with Modified Extended Latissimus Dorsi Muscular Flap Improve the Cosmetic and Functional Outcome among Patients with Breast Carcinoma?” World Journal of Surgery, Vol. 31, No. 6, 2007, pp. 1169-1177. doi:10.1007/s00268-007-9046-y

[12] H. M. Abdalla, M. A. Shalaan and F. A. Fouad, "Immediate Breast Reconstruction with Expander Assisted Latissimus Dorsi Flap after Skin Sparing Mastectomy,” Journal of the Egyptian National Cancer Institute, Vol.
18, No. 2, 2006, pp. 134-140.

[13] S. A. Salvin, S. J. Schnitt and R. B. Duda, "Skin-Sparing Mastectomy and Immediate Reconstruction: Oncologic Risks and Aesthetic Results in Patients with Early-Stage Breast Cancer," Plastic and Reconstructive Surgery, Vol. 102, No. 1, 1998, pp. 49-62. doi:10.1097/00006534-199807000-00008

[14] R. M. Simmons, S. K. Fish, L. Gayle, G. S. Trenta, A. Swistel, et al., "Local and Distant Recurrence Rates in Skin-Sparing Mastectomies Compared with Non-SkinSparing Mastectomies," Annals of Surgical Oncology, Vol. 6, No. 7, 1999, pp. 676-681. doi:10.1007/s10434-999-0676-1 